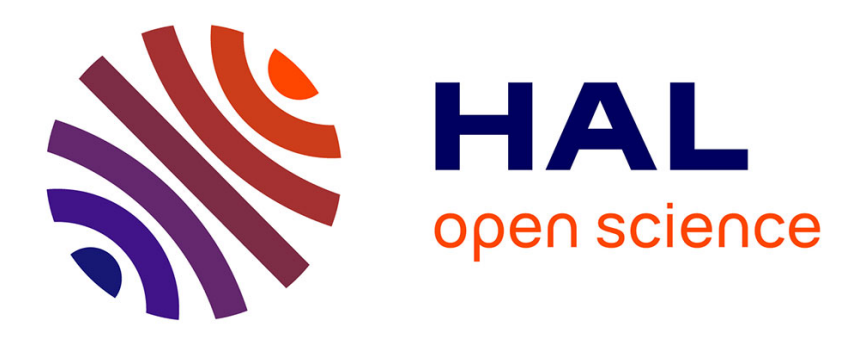

\title{
Performances et écriture de la critique
}

Jean-Luc Moriceau

\section{To cite this version:}

Jean-Luc Moriceau. Performances et écriture de la critique. Communication et organisation: perspectives critiques, Presses universitaires du Septentrion, pp.167 - 177, 2013, Capitalismes - éthique institutions, 978-2-7574-0430-0. hal-02402241

\section{HAL Id: hal-02402241 \\ https://hal.science/hal-02402241}

Submitted on 10 Dec 2019

HAL is a multi-disciplinary open access archive for the deposit and dissemination of scientific research documents, whether they are published or not. The documents may come from teaching and research institutions in France or abroad, or from public or private research centers.
L'archive ouverte pluridisciplinaire HAL, est destinée au dépôt et à la diffusion de documents scientifiques de niveau recherche, publiés ou non, émanant des établissements d'enseignement et de recherche français ou étrangers, des laboratoires publics ou privés. 
Fichier auteur : Une version plus complète a été publiée dans :

Thomas Heller, Romain Huet et Bénédicte Vidaillet, Communication \& Organisation :

perspectives critiques, Villeneuve d'Ascq, Presses Universitaire du Septentrion, pp. 167-177.

\title{
Performance et écriture de la critique
}

\author{
Jean-Luc MORICEAU ${ }^{1}$
}

Résumé : Posant la question : la critique de la communication ne s'autolimite-t-elle pas lorsqu'elle ne critique pas ses propres modes de communication?, ce texte explore les possibilités critiques offertes par les performances. Ces possibilités concernent l'intention de la communication, les formes de savoir tout comme les modalités (politiques) de la construction de la critique.

Mots-clefs : performance, performativité, critique

Le développement de la communication s'est accompagné d'un développement des théories de la communication et d'une critique de la communication. Celles-ci mettent par exemple sous examen les formes et présupposés des théorisations (Sfez, 1990), les utopies impliquées (Breton, 2004), les transformations induites dans la société (Leclerc, 1999) ou dans nos processus mentaux (De Kerckhove, 1997). Cependant la plupart des critiques académiques de la communication sont écrites sous la forme de communication académique mainstream. Nous voudrions nous demander: qu'apporterait à la critique de la communication d'autres formes d'expression et de transmission de la critique ? Ou plus polémiquement: la critique de la communication ne s'autolimite-t-elle pas lorsqu'elle ne critique pas ses propres modes de communication?

Les implications politiques du format académique de communication sont quelquefois dénoncées. Bourdieu (1982) par exemple en a analysé les limites : seuls seront publiés et écoutés ceux qui maîtrisent le langage académique et tous ses codes. Mais d'une manière plus générale, c'est la question de la représentation (dans son double sens : cognitif et politique) qui est interrogée. Les études qualitatives en sciences humaines ont été traversées au milieu des années 1980 par une crise de la représentation (cf. Denzin \& Lincoln, 2000). Débutée en ethnographie, la crise est partie de la reconnaissance de la place centrale de l'écriture, soulignant que l'étude consistait plus en une invention qu'en une représentation de la culture (Clifford \& Markus, 1986). Van Maanen (1995), de même que Geertz (1996), et dans un style magnifique, ont superbement montré que la théorie est dans le style. Ainsi l'écriture n'est pas seulement représentation, seconde et secondaire; elle est création,

\footnotetext{
${ }^{1}$ Institut Télécom/Télécom EM Research/Organization Studies, jean-luc.moriceau@it-sudparis.eu.
} 
elle peut même être un mode d'enquête (Richardson \& Saint Pierre, 2005). L'écriture est un travail (Solé, 2004), elle est la difficile expression d'une sensibilité, elle est la tentative de placer le lecteur dans un autre rapport au réel, pour qu'il l'appréhende avec une autre façon de le toucher, de le prendre, de le surprendre, de le comprendre. Toutes ses interrogations amènent ainsi à se demander : que pourrait une critique qui s'autoriserait à se communiquer dans d'autres modes d'écriture ?

Nous voudrions dans cette contribution interroger les possibilités critiques de ce qui est à la frontière de l'écriture et à la frontière de l'académique : les performances. Encore semble-t-il peu développées en France, les performance studies sont plus reconnues aux Etats-Unis. Leur intention affichée est justement de déconstruire un certain «apartheid des savoirs » (Conquergood, 2002, p.153), refusant d'opposer théorie et pratique, abstraction et incarnation, présentation et représentation. Elles mêlent, toujours selon Conquergood, critique, créativité et citoyenneté ; imagination, enquête (inquiry) et intervention; ou dit d'une autre manière encore : art, analyse et activisme (idem, p.152). Utiliser une performance dans le cadre d'un colloque académique n'est pas inédit, mais demeure très exceptionnel. Ceci peut conduire à des succès comme à des échecs cuisants et pose en tout cas un ensemble de questions quant à la communication académique.

Nous montrerons dans un premier temps les problématiques en jeu, ce que cherchent à accomplir et ce que remettent en question les performances académiques. Puis nous prendrons l'exemple d'une communication présentée à un colloque sous forme de performance. Ce qui nous amènera dans un troisième temps à présenter les possibilités critiques des performances pour un colloque de même que certaines questions décisives quant à son insertion dans le milieu académique.

\section{Visées de la performance}

Une performance vise plus à montrer qu'à démontrer, plus à présenter qu'à représenter (même si tous ces éléments sont présents). Plutôt par exemple que de parler à propos de la misère ouvrière il s'agit d'incarner l'ouvrier empêtré dans ses difficultés; produisant ainsi une communication composée davantage d'affects que de concepts, plus proche de l'expérience que du cognitif. Il s'agit à la fois d'être (being), de faire (doing) et de montrer le faire (showing doing) (Schechner, 2002); et non pas seulement d'analyser à distance ou de proposer une représentation. La performance est plus proche de l'événement, elle survient (Schechner, 2002), elle tente de produire ou de reproduire un morceau du réel. La vie quotidienne peut d'ailleurs être vue comme une suite de performances (cf. Goffman, 1996). Dans la performance académique, plutôt que de parler au nom de certaines personnes, il s'agit d'être un temps cette ou ces personne(s), d'incarner leur voix et leur condition et ainsi de donner à penser plutôt que dire ce qu'il faut en penser.

\section{Un autre partage du sensible}

Les performances apportent un autre «partage du sensible ». Rancière (2000) montre que ce qui nous est donné à voir et à savoir est organisé par un ensemble de codes et de conventions partagés qui 
assignent à chacun une place définie. Et pour lui cette mise en scène de l'esthétique est politique : «La politique porte sur ce qu'on voit et ce qu'on peut en dire, sur qui a la compétence pour voir et la qualité pour dire, sur les propriétés des espaces et les possibles du temps. » (p.14) ${ }^{2}$. Ainsi, avant même que de voir ou d'entendre une communication scientifique, nous savons quel est le rôle de chacun et c'est ce qui nous permet d'en comprendre le sens : les acteurs du terrain ont seulement été interrogés par le chercheur, le chercheur explique et théorise la situation, l'audience écoute et évalue le récit du chercheur.

Dans une performance, le chercheur est avant tout médiateur (même si sa médiation est non neutre et objet de réflexivité) : il vise à mettre en contact direct l'audience avec la parole et la condition des acteurs du terrain. Le spectateur est «émancipé », le sens ne lui est pas donné, il lui revient de le construire :

«L'émancipation, elle, commence quand on remet en question l'opposition entre regarder et agir, quand on comprend que les évidences qui structurent ainsi les rapports du dire, du voir et du faire appartiennent elles-mêmes à la structure de la domination et de la sujétion. Elle commence quand on comprend que regarder est aussi une action qui confirme ou transforme cette distribution des positions. Le spectateur aussi agit, comme l'élève ou le savant. Il observe, il sélectionne, il compare, il interprète. Il lie ce qu'il voit à bien d'autres choses qu'il a vues sur d'autres scènes, en d'autres sortes de lieux. Il compose son propre poème avec les éléments du poème en face de lui. Elle participe à la performance en la refaisant à sa manière, en se dérobant par exemple à l'énergie vitale que celle-ci est censée transmettre pour en faire une pure image et associer cette pure image à une histoire qu'elle a lue ou rêvée, vécue ou inventée. Ils sont à la fois ainsi des spectateurs distants et des interprètes actifs du spectacle qui leur est proposé. » (Rancière, 2008, p. 19)

\section{Une convocation des sens}

Le sens ainsi véhiculé, n'est pas clôt. Il n'est pas un sens unique. Il est donné à (multiples) interprétations. La représentation du sens est ainsi défiée à de nombreux niveaux. D’abord il s'agit de présenter l'objet d'étude en amont de sa fixation dans un sens attribué, l'objet d'étude valant par luimême avant d'être le signe d'un signifié. Ensuite parce que chaque geste, chaque parole peut évoquer de multiples références, par exemple une autre pièce ou un film, un tableau et ainsi être représentation de représentation (de représentation, etc.) ${ }^{3}$

Mais aussi il ne faut pas oublier que la performance est incarnée (embodied) et qu'ainsi son sens dépasse largement le sens des mots énoncés (comme les études sur la communication n'ont cessé de le répéter). La performance touche au sensible avant de dire un sens. Le sens ne se limite pas au sensé, mais embrasse en même temps le sensible, la sensation, la sensualité, la sensorialité, le sentimental et donc une plus grande palette des modalités du sens tel que Nancy (2009) les développe. Le

\footnotetext{
${ }^{2}$ La citation porte sur une esthétique à la base de la politique, qui ne vise pas spécifiquement les performances, mais que celles-ci permettent particulièrement d'illustrer.

${ }^{3}$ Sur ces deux écarts par rapport à la représentation moderne, voir Rancière (2000 et 2001).
} 
spectateur est affecté avant d'être informé. Le performeur ne transmet pas des signes, il incarne une condition.

\section{Une réactivation du désir}

Bernard Stiegler (2005) rappelle que la catharsis n'est pas seulement purgation des passions, mais qu'elle est aussi réactivation du désir. Le spectateur affecté n'est pas seulement le spectateur informé, celui qui acquiert une information ou un concept de plus, il est celui qui est transformé par ce à quoi il assiste. Stiegler dira que le spectateur est ainsi «individué » psychiquement et collectivement. Il devient ainsi individu (en même temps que les autres spectateurs). La performance «performe », c'està-dire qu'en disant elle agit (cf. Austin, 1970) ; elle provoque des effets sur les spectateurs. L'audience ne consomme pas de l'information ou des concepts (consommer au sens de Stiegler signifie acquérir sans devenir, sans en être transformé), elle assiste pour ne plus être tout à fait la même qu'auparavant.

\section{L'exemple d'une performance dans un colloque : le cocktail, ou de la guerre dans les affaires.}

Pour tenter d'illustrer et de réfléchir le potentiel critique des performances, nous avons présenté une communication sous forme de performance dans un colloque ${ }^{4}$. Cette communication se voulait doublement critique. D'abord parce que le récit était fort critique de la vie dans les organisations (montrant que les valeurs du conflit et de la guerre y sont érigées en principe et en pratiques) mais aussi, et surtout pour notre propos, parce que la forme de la présentation, sous forme de performance, voulait lancer le débat sur le potentiel critique de la communication par performance.

Quant au contenu, il s'agissait d'un texte rédigé par l'auteur qui reprenait la forme et le style du Banquet de Platon, livre qui est la base de la découverte, la transmission et l'éducation à la sagesse dans la tradition européenne et qui place Eros au centre de cet apprentissage. Le contexte avait été transposé dans une organisation d'aujourd'hui. Il s'agissait alors d'un cocktail où chacun se voyait demandé de porter un toast cette fois à Polémos... Ce n'est donc pas sous le signe et les valeurs d'Eros que notre monde fait d'organisations (Mintzberg, 2004) est éduqué et prend pour modèle mais bel et bien Polémos. Ce thème était l'intention du texte, mais celle-ci n'était jamais explicitée ou discutée ; il revenait aux spectateurs de faire leur propre « poème » à partir de ce à quoi ils assistaient.

Le texte reprenait à l'identique la structure narrative du texte de Platon, il en reprenait directement certaines phrases et son style. De même les théories d'auteurs célèbres étaient portées par certains personnages sans être commentés, explicités ou analysés, le texte voulait ainsi avoir certains éléments de polyphonie sans être dirigé ou cadré par la pensée de son auteur.

\footnotetext{
${ }^{4}$ Il s'agit du colloque « Communications-organisations et pensées critiques » organisé par la SFIC à Lille en 2011. La même performance avait déjà été présentée au colloque «Eros et Polémos dans les organisations » à l'Istec en 2009.
} 
La performance a été accomplie par l'auteur et deux autres participants volontaires, avec un minimum de mise en scène, sans avertir explicitement l'audience du but de mise en débat du potentiel critique des performances à la recherche en communication.

Pour rendre plus concret le récit de l'expérience, nous proposons un résumé du texte de la performance.

Le narrateur se félicite d'avoir enfin pu rencontrer un témoin d'un cocktail dont il avait juste entendu parler en la personne de L. Il lui demande de raconter ce qui s'était passé. L explique qu'à la suite du succès d'un contrat, lors du cocktail, le directeur avait demandé aux collaborateurs autour de lui de porter un toast à Polemos.

La première à parler, Eve Smith, DRH, affirme que ce qui permet les plus hautes performances est la lutte pour survivre. Dans un discours empreint de darwinisme social, prenant des exemples mimant des travaux tels ceux de Nelson et Winter (1982), Hannan et Freeman (1977) ou même Weick (2000), elle montre comment dans ses pratiques elle favorise une certaine sélection naturelle et contraint chaque salarié à surpasser les autres car emploi, entreprise ou même sens partagé sont menacés par un environnement concurrentiel.

Philippo Zanias, représentant syndical rétorque qu'il faut distinguer entre les combats et en appeler à Polemos seulement pour les causes favorisant l'emploi et la solidarité. Dans une logique marxiste, il souligne que c'est effectivement la lutte et la contradiction qui font avancer le système et s'il faut encourager le meilleur de chacun c'est dans l'horizon d'un avenir apaisé et partagé.

Le troisième, Stéphane d'Aristo, directeur du marketing, remonte à un passé de l'entreprise où celle-ci ne faisait qu'une, solidaire et unie, androgyne. Et que, à la suite d'évaluations individualisées, qui ont affecté la subjectivité de chacun, c'est depuis la lutte pour la reconnaissance qui les anime tous. Chacun veut capter la totalité de la reconnaissance. On retrouve dans son propos des analyses proches de Ricoeur (2004), Honneth (2000) ou Roberts (1991).

Arrive ensuite Teussok. Agaçant, Teussok entre en dialogue avec Stéphane d'Aristo, et le conduit maïeutiquement par toute une série de questions. Il poursuit en rapportant le récit de Moneytime, qui expliquait que Polemos était fils de Position et Mouvement, cherchant toujours à défendre ses places et son ordre, mais en même temps à se réinventer et repositionner. Il montre comment la sagesse dans les affaires se construit progressivement, débutant par la contemplation des projets en général, pour descendre par étape, dans les discours et raisonnements, dans les règles et procédures jusque dans les têtes et les corps.

Entre en scène Alcide Biad, ancien salarié de l'entreprise, ivre. Comme souffrant du syndrome de Stockholm, il fut jadis amoureux de l'entreprise qui pourtant le kidnappait de ses proches. Il décrit un rapport sadomasochiste avec la discipline et l'engagement pour l'entreprise, proche des descriptions de Foucault ou des analyses de Pesqueux (2009) ou Gherardi (1995), et illustrant la proposition de Teussok. Jusqu'au jour où pour plaire encore davantage il démissionna, perdant alors tout mais gardant une gratitude pour son bourreau. 
Là-dessus L insiste après du narrateur pour qu'il écrive cette histoire dans un livre à large lectorat, racontant ce qui s'est passé et lui accordant une juste place. Secrétaire du directeur elle avait été écartée par une plus jeune et avait pris une revanche le soir de ce cocktail mais cherchait de la reconnaissance. Pourtant le narrateur, faisant semblant de la suivre, se promet d'en faire plutôt un article académique, lui-même pris dans un jeu universitaire tout aussi transi par Polémos.

\section{Les possibilités critiques des performances}

Toutefois, d'une certaine manière, dans cette communication le récit, le contenu de ce qui était présenté, était secondaire. C'était la forme, le fait de mettre en évidence la dimension performative qui était le but. Le contenu n'était pas exposé mais performé, incarné, proposé à l'interprétation ; c'était ainsi en même temps, à un niveau supérieur, la dimension performative qui était performée, incarnée et proposée à l'interprétation et à la discussion.

Toute communication dans un colloque est une performance, ou du moins contient toute une dimension performative, qui est souvent niée ou refoulée. Mettre celle-ci en évidence est une manière de proposer un ensemble de possibilités pour communiquer la critique et par ailleurs de critiquer un certain mode de communication dans les colloques académiques.

Tout d'abord la question n'est pas de savoir si la performance est vraie ou fausse, elle n'est pas en premier lieu celle de l'exactitude ou de l'adéquation. Certains éléments de performance sont parfois introduits dans le discours scientifique mais dans un but rhétorique. Il s'agit alors de mieux convaincre que ses idées sont justes. Mais la rhétorique n'est pas la performativité. La question pour une performance est de savoir si « elle marche », c'est-à-dire si elle réussit à produire des effets, si quelque chose est changé dans les perceptions et la compréhension d'un phénomène, si quelque chose a été donné à penser et que cette proposition a engendré une évolution des conceptions. Stiegler dirait s'il y a eu de l'individuation. En termes de performance studies on se demandera s'il y eu « félicité », c'està-dire si le dire, la communication, a eu un effet sur le réel.

Ensuite, les performances peuvent être utiles à la critique si leur intention n'est pas de transmettre autrement la même chose, le même type de savoir (cognitif, conceptuel). Leur but serait plutôt de transmettre une autre forme de savoir, ce qui justement ne peut être véhiculé par la forme habituelle des communications académiques. Ici, quelque chose est transmis par le corps (les postures, la voix, les expressions, etc.), par les affects, par la mise en scène (mouvements, éléments de décor, lumière, costumes...). Elles font écho à tout un ensemble de «tournants» qui animent actuellement les sciences sociales : turn to embodiement, turn to affect, turn to practice, etc. Elles sont particulièrement adaptées aux recherches concernant l'identité ou l'injustice sociale, à la critique postcoloniale ou de genre. En effet le sens de ce qui est transmis est enrichi, altéré, renforcé, nuancé, par ces éléments performatifs, qui peuvent conserver et donner à penser la complexité, les contradictions, les enjeux éthiques de l'objet d'étude. Comme nous l'avons dit plus haut, la performance s'oppose ou offre la possibilité de jouer avec la représentation. Les communications académiques ont le plus couramment 
pour but de proposer et discuter une (ou des) représentation(s). La performance ouvre la représentation d'un côté à un contact plus direct avec le terrain étudié, et d'un autre à un jeu de multiples références sur des références (cf. Rancière, 2000). Il est également plus facile d'introduire certaines modalités de discours comme de l'ironie, de l'authenticité, de la manipulation, une sauvegarde de la face... ou de soupçonner que certains dires ne devraient pas être pris pour argent comptant (ce qui est si difficile avec les analyses de verbatims).

Enfin, les performances participent d'une autre forme de construction du savoir. D'une part car elles manifestent un autre partage du sensible. Ce n'est plus le chercheur qui construit une représentation du terrain et tâche de convaincre les autres chercheurs. Le savoir, ou plutôt les multiples possibilités de savoir, sont co-construites par les acteurs du terrain, le chercheur et l'audience. Les acteurs du terrain peuvent participer de manière plus ou moins forte à la performance, en exprimant leur voix, contribuant à l'écriture du scénario jusqu'à interpréter (et discuter) leur propre rôle (cf. Denzin, 2003). Il est de même plusieurs manières d'impliquer les spectateurs (de la création d'un événement durant le colloque jusqu'aux pratiques du Living Theatre). D'autre part, les performances donnent plus de possibilités à une certaine polyphonie (Boje, 2011, Czarniawska, 2005). Les différentes voix des parties-prenantes peuvent être présentées sans qu'il soit nécessaire de les hiérarchiser, de les traduire ou de les subsumer sous la voix de l'auteur de la communication. L'auteur de la performance est d'emblée un auteur collectif (cf. sur ce point Derrida, 1990).

Dans l'exemple de la performance interprétée dans le colloque, des contraintes d'organisation ont imposé que la mise en scène était réduite au minimum et le travail des personnages sommaire. Toutefois les questions qui suivirent, de même que plusieurs commentaires adressés après, certains passionnés, ont tous portés non sur le contenu mais sur la performativité. Ce qui laisse penser que la performance avait produit un certain effet. La félicité n'est pas mesurable mais on peut supposer qu'elle était mitigée - ce qui apporte plusieurs questions sur la pratique de performances en milieu académique.

\section{Questions sur l'insertion de performances dans le milieu académique}

En effet le recours à des fins critiques de performances dans les colloques académiques ne peut pas échapper à son tour à une certaine critique.

Tout d'abord, vient la question de savoir comment évaluer une performance. Sa performativité, son impact, ne peut être évalué (réduite) par des facteurs d'impact classiques. Elle ne peut pas être non plus évaluée selon les critères courants de validité et de fiabilité (critères qui s'appliquent plutôt aux représentations même si ces dimensions en sauraient être complètement absentes). L'évaluation devrait au moins pour une part comporter des critères propres aux disciplines artistiques auxquels les universitaires sont rarement formés. Elle devrait également inclure une inquiétude concernant la représentation politique des acteurs. L'évaluation devrait enfin s'apprécier en regard de l'intention 
ayant présidée à son effectuation (quel mouvement, quel effet la performance se propose-t-elle d'accomplir?)

Ensuite comment les performances peuvent-elles s'insérer dans le jeu des publications, jeu décisif pour les carrières académiques? D'une part, les performances ne rentrent pas dans le format classique des revues et sont généralement rejetées d'emblée. Mais, d'autre part, la performance ne saurait être réduite à son texte. Une captation vidéo serait évidemment concevable, mais poserait des questions autour de la construction de l'image et surtout perdrait tout un ensemble d'élément de présence, de direct et d'immédiateté qui sont constitutifs de la performance.

Enfin, il nous faudrait apprendre à bâtir des performances efficaces. Rien n'est plus vain qu'une performance qui ne «marche » pas. Toute une recherche et de nombreuses tentatives seront sans doute nécessaires pour apprendre à construire des performances efficaces et adaptées au milieu académique. Par ailleurs un débat peut être ouvert de savoir si la performance doit se suffire par elle-même ou si elle doit être accompagnée par une explicitation et une réflexion par les performers et/ou le chercheur.

\section{Conclusion}

Nous espérons avoir montré que les performances peuvent apporter un ensemble de possibilités pour la critique en sciences de la communication ou des organisations. Elles montrent également en creux les conventions et limites des modes les plus courants de communication de la critique. Il reste cependant tout un apprentissage à engager pour apprendre comment bâtir des performances efficaces et adaptées à la critique souhaitée et pour les insérer dans le paysage institutionnel académique.

\section{Bibliographie}

Austin J.L., 1970, Quand dire c'est faire, Seuil.

Boje D. 2011. Storytelling organizations, Sage publications.

Bourdieu P. 1982. Ce que parler veut dire: L'économie des échanges linguistiques, Arthème Fayard.

Breton P. 2004. L'utopie de la communication : Le mythe du village planétaire, La Découverte.

Conquergood D. 2002. Performance Studies: Intervention and Radical Research, The Drama Review, Vol.46, nº 2 , p.145-156.

Czarniawska B. 2005. De la polyphonie dans l'analyse des organisations, Revue française de gestion, vol. $6, \mathrm{n}^{\circ} 159$, pp. 359-371.

De Kerckhove D. 1997. The Skin of Culture, Kogan Page.

Denzin N.K. 2003. Performance Ethnography: Critical Pedagogy and the Politics of Culture. Sage.

Denzin N.K. and Lincoln Y.S. 2000. The Discipline and Practice of Qualitative Research, in Denzin NK.K \& Lincoln Y.S. Handbook of Qualitative Research, $2^{\text {nd }}$ ed., Sage, pp. 1-28.

Derrida J. 1990. Limited inc. Gallilée.

Geertz C. 1996. Ici et Là-bas: l'anthropologue comme auteur, Métailié.

Gherardi S.1995. Gender, symbolism and organizational cultures, Sage. 
Hannan M.T. \& Freeman J. 1977. The Population Ecology of Organizations. American Journal of Sociology, vol 82, n5, pp. 929-964.

Honneth A. 2000. La lutte pour la reconnaissance, Le Cerf.

Leclerc G. 1999. La société de communication: Une approche sociologique et critique, Presses Universitaires de France.

Mintzberg H. 2004. Le Management : Voyage au centre des organisations, Economica.

Nancy J-L. 2009. Faire sens, texte présenté au congrès Making Sense, Cambridge, Septembre.

Pesqueux Y. 2009. Un modèle sadomasochiste de l'organisation ?, Management \& Avenir, vol. 29, $\mathrm{n}^{\circ}$, pp. 17-36.

Rancière J. 2000. Le Partage du sensible : Esthétique et politique, La Fabrique.

Rancière J. 2001. La Fable cinématographique. Le Seuil.

Rancière J. 2008. Le Spectateur émancipé. La Fabrique.

Richardson L. \& St. Pierre E. 2005. Writing: A Method of Inquiry, in N. Denzin and Y. Lincoln (sous

la direction de) Handbook of Qualitative Research (Third Edition), Sage, pp. 959-978.

Ricoeur P. 2004. Parcours de la reconnaissance, trois études, Stock.

Roberts J., 1991, The Possibilities of Accountability, Accounting, Organizations and Society, Vol. 16 Issue 4, pp. 355-368.

Schechner R. 2002. Performance Studies: An introduction. Routledge.

Sfez L. 1990. Critique de la communication, Seuil.

Stiegler B. 2005. Grand Témoin, in Mises en scène du monde, Les Solitaires Intempestifs.

Van Maanen J. 1995. Style as theory, Organization Science, vol. 6, n ${ }^{\circ}$, pp. 133-142.

Weick Karl E., 1980. The Social Psychology of Organizing 2e éd., McGraw-Hill Publishing Co. 